\title{
Use of Interventional Neuroradiology Devices (Solitaire AB and Cascade Net) in the Treatment of Complex Renal Aneurysms through Stent-Assisted Coil Embolisation
}

\author{
Eva Pampín* ${ }^{\circ}$, Fernando López, Francisco Javier Maynar, Rebeca Bastida, Amaya Iturralde \\ Deparment of Interventional Radiology of HUA, Spain \\ Email: evapam@hotmail.com
}

How to cite this paper: Pampín, E., López, F., Maynar, F.J., Bastida, R. and Iturralde, A. (2021) Use of Interventional Neuroradiology Devices (Solitaire AB and Cascade Net) in the Treatment of Complex Renal Aneurysms through Stent-Assisted Coil Embolisation. Surgical Science, 12, 365-373. https://doi.org/10.4236/ss.2021.1211038

Received: October 23, 2021

Accepted: November 22, 2021

Published: November 25, 2021

Copyright $\odot 2021$ by author(s) and Scientific Research Publishing Inc. This work is licensed under the Creative Commons Attribution International License (CC BY 4.0).

http://creativecommons.org/licenses/by/4.0/

\begin{abstract}
Introduction: The risk of rupture of true renal artery aneurysms is low but when they are bigger than $2-2.5 \mathrm{~cm}$ it increases significantly, making treatment essential. The need to use alternatives to conventional techniques in order to avoid predictable complications as coil migration is mandatory. Discussion: Routinely-used techniques in interventional neuroradiology such as flow diverters or those assisted with an occlusion balloon or stent have are suitable alternatives for complex aneurysms. Conclusion: Interventional neuroradiology devices such as the Cascade Net stent (Perflow Medical and Grupo Logsa) and Solitaire AB stent retriever (Medtronic) are valid and safe options. We describe the technique of such devices.
\end{abstract}

\section{Keywords}

Embolisation, Aneurysm, Renal, Technique, Devices, Neuroradiology

\section{Introduction}

True renal artery aneurysms have a prevalence of less than $1 \%$.

The risk of rupture is low but when they are bigger than $2-2.5 \mathrm{~cm}$ it increases significantly, making treatment essential [1].

The endovascular treatment of wide neck aneurysms or aneurysms located in the bifurcations of the renal artery is a challenge for conventional endovascular techniques (simple coil embolisation) due to the risk of coil migration. The use of stents/grafts is another valid but less popular option due to the increased risk of areas of ischaemia in this renal location (bifurcations) [2]. 
Routinely-used techniques in interventional neuroradiology such as flow diverters or those assisted with an occlusion balloon or stent have proven to be suitable alternatives for complex aneurysms in the visceral arteries [3].

The following section describes the technique carried out for the assisted embolisation of a complex aneurysm in the bifurcation of the right renal artery using common interventional neuroradiology devices such as the Cascade Net stent (Perflow Medical and Grupo Logsa) and Solitaire AB stent retriever (Medtronic).

\section{Clinical Case}

54-year-old patient with pain in right side for approximately one year, has a CT angiography which shows an aneurysm in the right renal artery. A general physical examination reveals untreated high blood pressure $(156 / 103 \mathrm{mmHg})$ and creatinine $1.3 \mathrm{mg} / \mathrm{dl}$ (previously $0.8 \mathrm{md} / \mathrm{dl}$ ). Preventive treatment started with ASA (Adiro 100) and check-ups every 3 months via a CT angiography. After a review by a multi-disciplinary team comprising Interventional Radiology and Vascular Surgery, it is finally decided to carry out an embolisation.

Controlled sedation was carried out by the intensive care team.

Peri-procedural thrombogenic prevention with AGGRASTAT (Tirofiban) in continuous infusion at a dose of $40 \mathrm{ml} / \mathrm{hr}$ for $30 \mathrm{~min}$ and then $10 \mathrm{ml} / \mathrm{hr}$ as prescribed.

After administering local anaesthetic to the right inguinal region, a micropuncture technique is used to access the right common femoral artery before inserting the $4 \mathrm{~F}$ introducer (Terumo).

A cobra $24 \mathrm{~F}$ catheter (Cook) is used to create a series of diagnostic angiograms in different projections from the renal artery and selective angiograms of each of the main branches, paying attention not only to arterial vascularisation but also to the distribution of the renal infusion for subsequent decisions.

This is a complex renal aneurysm affecting the main division of the right renal artery. It extends slightly into the anterior segmental branch of said division with a wide neck $(10 \mathrm{~mm})$ while the posterior segmental branch exits from the aneurysm itself, very close to the division (Figure 1).

The introducer is switched to the Destination 7F $45 \mathrm{~cm}$ renal curve introducer (Terumo) which is pushed in a proximal direction in the right renal artery (Figure 2).

A Rebar 180.021 (Ev3) micro catheter is inserted into the anterior segmental branch and a Prowler Select plus 0.021 (Cardiva) micro catheter into the posterior segmental branch. A Lantern 0.025 (Penumbra) embolisation micro catheter is also inserted into the aneurysmal sac. These three catheters are parallel to each other inside the 7F introducer (Figure 3).

The Solitaire $6 \times 30 \mathrm{~mm}$ (Medtronic) endoprosthesis is inserted through the first micro catheter, the Rebar $18(\mathrm{eV} 3)$, in order to preserve the anterior branch.

The Cascade Net (Perflow-grupo Logsa) remodelling system for 5 - $6 \mathrm{~mm}$ 


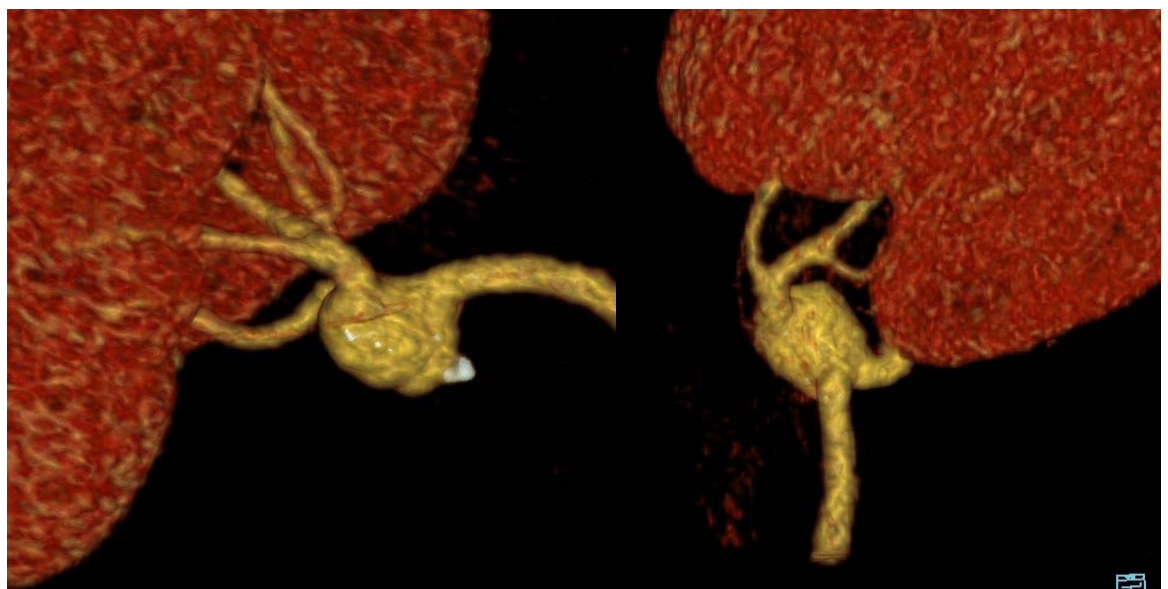

Figure 1. The complex renal aneurysm affects the main division of the right renal artery.

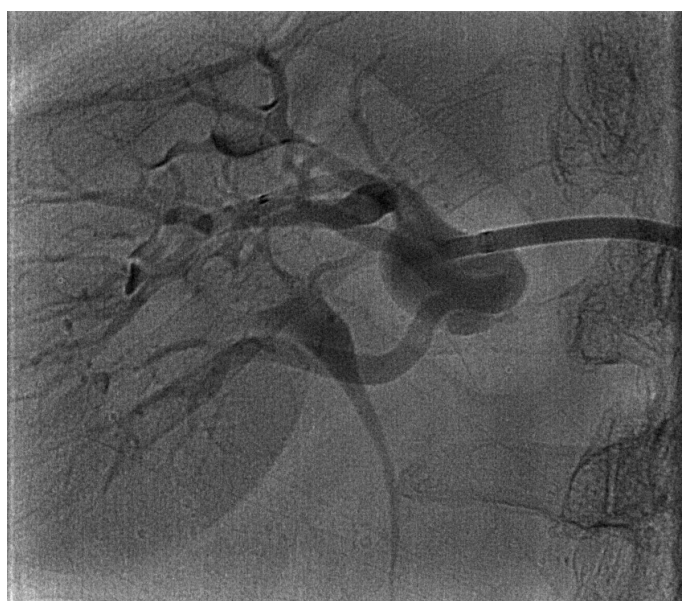

Figure 2. Destination 7F $45 \mathrm{~cm}$ renal curve introducer (Terumo) is pushed in the right renal artery.

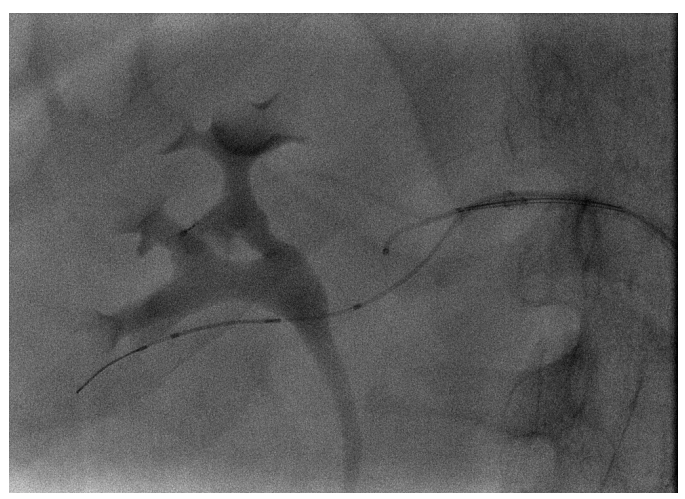

Figure 3. Three microcatheters are parallel to each other inside the $7 \mathrm{~F}$ introducer.

vessels is inserted through the second catheter, the Prowler Select Plus (Cardiva), in order to preserve the posterior branch.

After inserting the third micro catheter, Lantern (Penumbra), into the aneu- 
rysmal sac, the embolisation is carried out using $14 \times 60 \mathrm{~mm}$ standard Ruby coil micro coils for framing (Penumbra) and the sac then filled with $12 \times 40$ and $8 \times$ 60 soft Ruby coil micro coils (Penumbra) (Figure 4 and Figure 5).

After filling and packing the aneurysmal sac, we proceed to remove the Solitaire $\mathrm{AB}$ (Medtronic) device. While doing so we observe that one of the spirals of a coil is being pulled towards the main renal artery, so a decision is made to release it and leave it in the anterior branch permanently.

There were no problems withdrawing the Cascade Net system (Perflow-Logsa group).

The final follow-up angiogram confirms the complete obliteration of the aneurysmal sac with stent permeability and, therefore, the preservation of both of the division branches, as well as the renal parenchyma (Figure 6).

During the procedure, the patient remained haemodynamically stable with a brief period of bradycardia and hypotension at the start as a result of a vagal response which was resolved with $1 \mathrm{mg}$ IV of atropine and fluid therapy.

There were no other periprocedural complications and the follow-up arteriogram after 24 hours of treatment was completely normal (Figure 7).

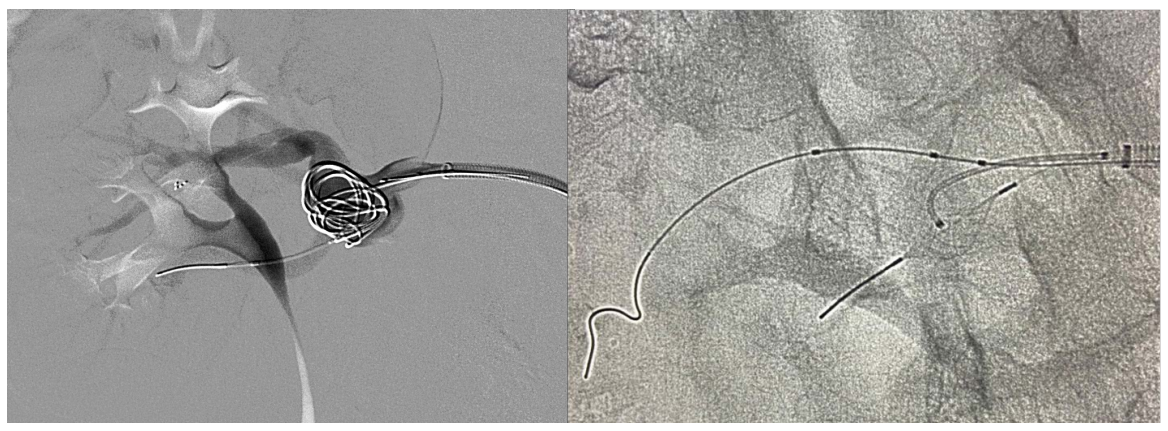

Figure 4. The Cascade Net (Perflow-grupo Logsa) remodelling system is inserted in order to preserve the posterior branch during the embolisation.

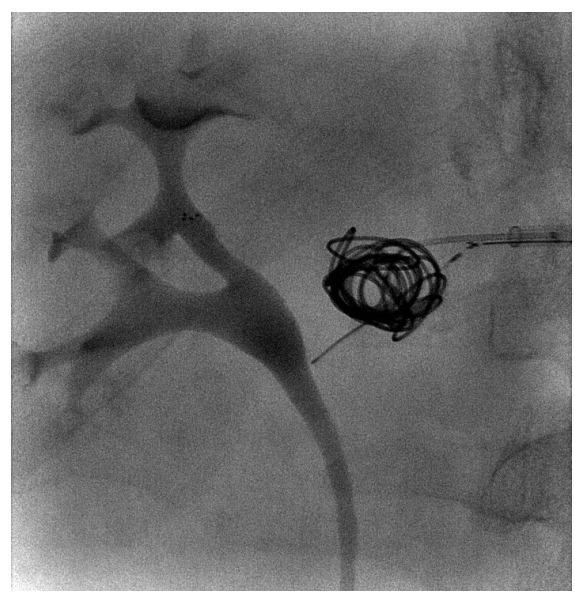

Figure 5. Embolisation is carried out using standard Ruby coil micro coils for framing (Penumbra) and the sac then filled with soft Ruby coil micro coils (Penumbra). 


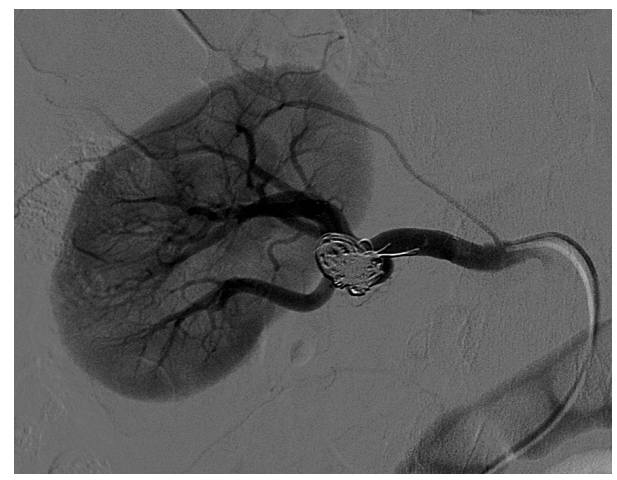

Figure 6. The final follow-up angiogram confirms the complete obliteration of the aneurysmal sac with preservation of both of the division branches, as well as the renal parenchyma.

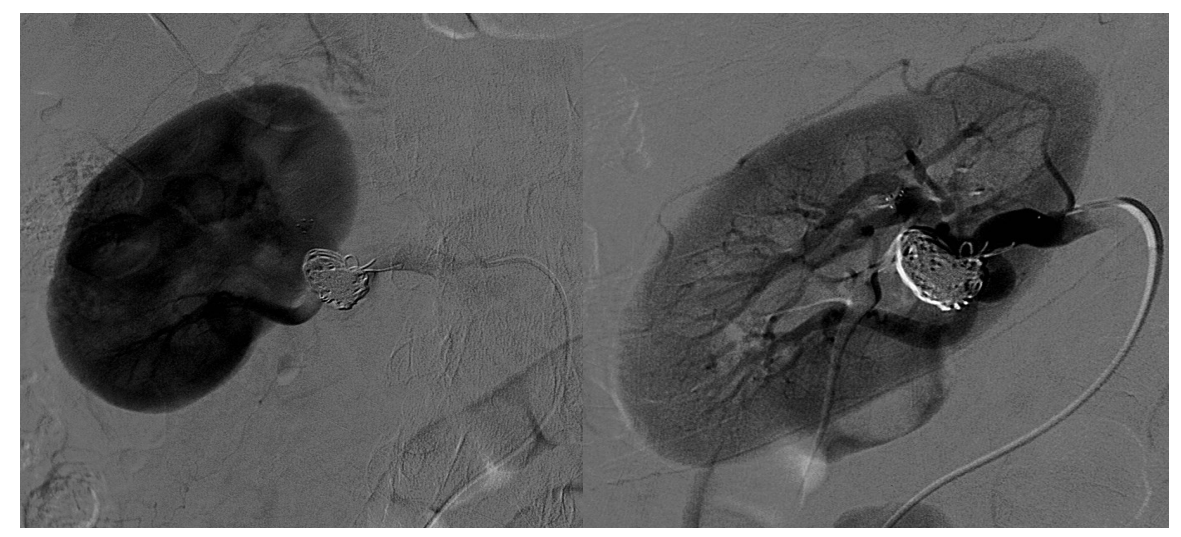

(a)

(b)

Figure 7. (a) Angiogram shows the preservation of the renal parenchyma. (b) The follow-up arteriogram after 24 hours of treatment without periprocedural complications.

The patient was discharged after 5 days without complications and prescribed dual anti-platelet therapy with DUOPLAVIN (Clopidogrel $75 \mathrm{mg} / \mathrm{ASA}$ $100 \mathrm{mg}$ ) for at least three months. The follow-up doppler echocardiogram after one month, as well as the follow-up CT angiogram after 4 months, did not show any changes and the permeability of the entire vascular tree and renal infusion was complete (Figure 8 and Figure 9).

\section{Discussion}

True renal artery aneurysms make up $22 \%$ - 25\% of all visceral aneurysms with an estimated incidence of $0.1 \%-1 \%$ in the general population, increasing to around $2.5 \%$ in patients with high blood pressure [1].

The risk of rupture is low (5.6\%) but it increases significantly during pregnancy $(80 \%)$ and as the aneurysm increases in size, so those with a sac larger than $2-2.5 \mathrm{~cm}$ or where there is evidence of growth between scans must be treated. Pseudoaneurysms or false aneurysms must be treated regardless of their 


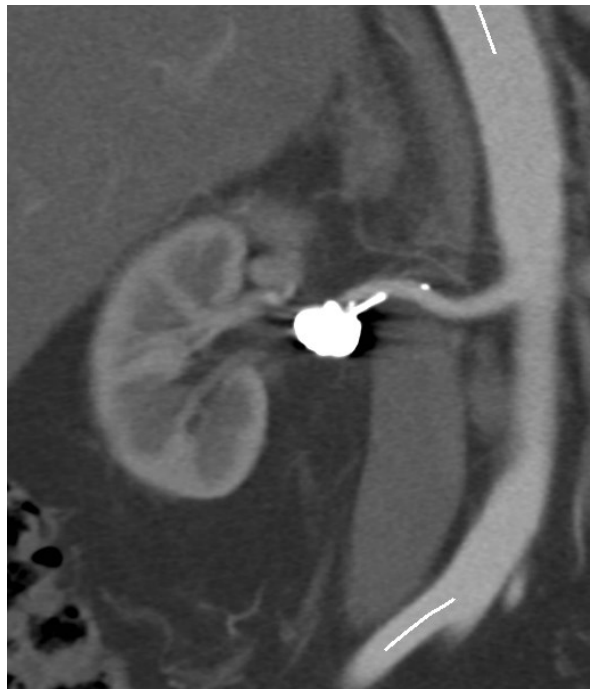

Figure 8. Follow-up CT angiogram after 4 months showed complete renal perfusion.

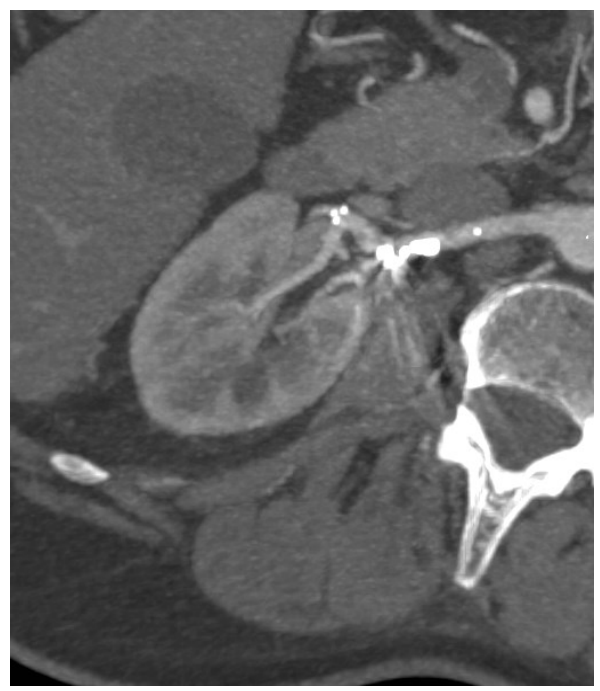

Figure 9. Follow-up CT angiogram after 4 months showed permeability of the entire vascular tree.

size due to the high risk of associated rupture [4].

There are no significant differences between endovascular and surgical treatment in terms of post-operation mortality or complications within 30 days, although the average hospital stay is significantly longer for those recovering from surgery (14 vs. 4 days). In urgent rupture cases, endovascular treatment has a lower mortality rate $(2.7 \%$ vs. $23.7 \%)$ but the rate of re-permeabilisation is higher (7.6\%) [5].

The endovascular treatment of wide neck aneurysms or aneurysms located in the bifurcations of the renal artery is a challenge for conventional endovascular techniques (simple coil embolisation) due to the risk of coil migration [6].

The most important factors when choosing between techniques are the size of 
the aneurysm and the diameter of its neck, as well as the neck-to-sac ratio. A wide neck $(\geq 4 \mathrm{~mm})$ or a neck-to-sac ratio $\geq 2$ in narrow necks and $<2$ in wide necks indicate the need to use alternatives to conventional techniques in order to avoid predictable complications.

The use of stents/grafts is another valid option within conventional techniques, although it has not proven to be the most appropriate for these sites, unlike the main renal artery, because the branches to the side of the branch to be treated are excluded and covered by the stent, which may lead to areas of renal ischaemia. Furthermore, the high profile of covered stents and difficulty inserting them into narrow and curved branches such as in this case make their devices to be avoided in this area in routine practice [2].

Commonly used techniques in interventional neuroradiology such as flow diverters or embolisation techniques assisted by an occlusion balloon or stent retriever have proven to be suitable alternatives for complex aneurysms in the visceral arteries.

Flow diverter stents appeared and were designed specifically for maintaining laminar flow in the main artery and its branches while simultaneously decreasing the flow rate in the aneurysm, favouring sac thrombosis (6). Despite being devices created for intracranial use, their recent application in aneurysms in any part of the body has had good results in terms of stent permeability and reducing the volume of the aneurysmal sac [7].

Occlusion balloon-assisted coil embolisation is a standardised technique for treating wide neck intracranial aneurysms. It is used when the first framing coil feels unstable in the aneurysm, in which case inflating the balloon with careful control of the inflation timing gives the framing coil stability, deflating it before releasing it, checking that the coil does not prolapse into the main vessel. Inflation times must be closely monitored in order to avoid sustained hypoperfusion and subsequent ischaemia. Studies have also been carried out on embolisation with liquid agents \pm coils as a variant of the common balloon-assisted technique [8].

Stent-assisted coil embolisation is another very useful technique for wide neck and geometrically complex aneurysms, as the stent acts as a reinforcement that enables coils to be deposited, avoiding them becoming herniated in the main vessel, with the option of leaving it in the artery permanently [9].

In this case, due to the location and morphology of the aneurysm, stentassisted coiling was the technique chosen to immediately occlude the aneurysm without coil herniation or unwanted embolisations while preserving renal flow. This is a complex saccular/fusiform renal aneurysm affecting the main division of the right renal artery. It extends slightly into the anterior segmental branch of said division with a wide neck $(10 \mathrm{~mm})$ while the posterior segmental branch extends from the aneurysm itself, very close to the division (Figure 1). Releasing the framing coils through the microcatheter in the aneurysmal sac was very unstable with a tendency for herniation in the anterior and posterior branches of 
the division, making framing impossible without a stabilising device (Figure 4 and Figure 5).

The covered stent option was discounted because of the curved and complicated nature of the anatomy, with a risk of renal ischaemia due to the exclusion of unwanted branches. The use of a flow diverter would have been an alternative but a lack of experience with these normally intracranial devices and their cost (off-label in peripheral) meant they were left as a second option in case the technique chosen as a first option failed (assisted coiling with stent retriever). Similarly, the covered stent and flow diverter were used to try to avoid mandatory dual anti-platelet therapy at the start.

In our case, the new non-occlusive device Cascade Net (Perflow Medical and Grupo Logsa) was used due to it being densely braided in order to give the coils stability without blocking the blood flow, keeping the device open for more than two hours in the posterior branch with continuous blood flow before removing it without problems.

The Solitaire $\mathrm{AB}$ (Medtronic) was used in the anterior branch at the same time. Although the original plan was not to leave it there permanently, the movement of one of the spirals of the packed coils towards the main renal artery during one of the actions to reposition it meant it had to be left in the anterior branch permanently to preserve the flow. Following that there were no problems or peri-procedural thromboembolic events during the first 24 hours or following 4 days before discharge.

\section{Conclusion}

The Solitaire AB and Cascade Net intracranial devices routinely used in interventional neuroradiology are valid and safe options to use as devices in the endovascular treatment of true complex aneurysms of the renal arteries as part of coil-assisted embolisation techniques, preserving renal flow and with a minimal complication and peri-procedural mortality index [7].

\section{Conflicts of Interest}

The authors declare no conflicts of interest regarding the publication of this paper.

\section{References}

[1] Elaassar, O., et al. (2011) Endovascular Techniques for the Treatment of Renal Artery Aneurysms. Cardio Vascular and Interventional Radiology, 34, 926-935. https://doi.org/10.1007/s00270-011-0127-9

[2] Rabuffi, P., Bruni, A., Antonuccio, E.G.M., Ambrogi, C. and Vagnarelli, S. (2020) Treatment of Visceral Artery Aneurysms and Pseudoaneurysms with the Use of Cerebral Flow Diverting Stents: Initial Experience. CVIR Endovascular, 3, Article No. 48. https://doi.org/10.1186/s42155-020-00137-y

[3] Chung, R., Touska, P., Morgan, R. and Anna-Maria, B. (2016) Endovascular Management of True Renal Arterial Aneurysms: Results from a Single Centre. Cardi- 
o Vascular and Interventional Radiology, 39, 36-43.

[4] Wojtaszek, M. (2013) Managing Visceral Artery Aneurism. Current Endovascular Techniques and Technologies for Treating This Increasingly Encountered Presentation. Endovascular Today, 77-81.

[5] Ccochenneca, F., Riga, C.V., Allaire, E., et al. (2011) Contemporary Management of Splanchnic and Renal Artery Aneurysms: Results of Endovascular Compared with Open Surgery from Two European Centers. European Journal of Vascular and Endovascular Surgery, 42, 340-346. https://doi.org/10.1016/j.ejvs.2011.04.033

[6] Elda, G., Erogan, E., Peynircioglu, B., et al. (2019) Endovascular Treatment of True Renal Artery Aneurysms: A Single Center Experience. Diagnostic and Interventional Radiology, 25, 62-70. https://doi.org/10.5152/dir.2018.17354

[7] Hardman, R.L., Taussky, P., Kim, R. and O’Hara, R.G. (2015) Post-Transplant Hepatic Artery Pseudoaneurysm Treated with the Pipeline Flow-Diverting Stent. Cardio Vascular and Interventional Radiology, 38, 1043-1046. https://doi.org/10.1007/s00270-015-1115-2

[8] Das, J.P., Asadi, H., Kok, H.K., Phelan, E., O’Hare, A. and Lee, M.J. (2018) Balloon-Assisted Coil Embolization (BACE) of Wide-Necked Renal Artery Aneurysm Using the Intracranial Scepter C Compliant Occlusion Balloon Catheter. CVIR Endovascular, 1, Article No. 12. https://doi.org/10.1186/s42155-018-0018-0

[9] Manninen, H.I., Berg, M. and Vanninen, R.L. (2008) Stent-Assisted Coil Embolization of Wide-Necked Renal Artery Bifurcation Aneurysms. Journal of Vascular and Interventional Radiology, 19, 487-492. https://doi.org/10.1016/j.jvir.2007.10.026 